\title{
Outage Probability in Opportunity Relaying Cooperative Diversity System over Rayleigh Fading Channels with Interferes
}

\author{
Meirong $\mathrm{He}^{1, \mathrm{a}^{*}}$ and Chunling Zhang ${ }^{1, \mathrm{~b}}$ \\ ${ }^{1}$ Northwest University for Nationalities, Lanzhou, Gansu, 730030, China \\ ahmr@xbmu.edu.cn, ${ }^{b} 383045799 @ q q . c o m$ \\ *The corresponding author
}

Keywords: Cooperative diversity; Outage probability; Interferes; Relays

\begin{abstract}
In this paper, we investigate the outage probability in opportunity relaying system with multiple unequal-power Rayleigh interferes at both relay and destination, the closed form expression for the outage probability is derived. Through simulation, it shows that the whole system outage performance can be improved effectively with multiple selected relays .
\end{abstract}

\section{Introduction}

Cooperative diversity communication has been established as a promising technique to combating fading, improving high-data coverage and reliability required for high transmission in wireless communication. At present, the research of cooperative diversity technique shows that the wireless system's performance can be improved tremendously. However, the cooperative diversity networks will be subjected to channel interference in practice, so channel interference is a important factor that can affect the performance significantly. Recently, channel interference has been consideration in some works. In[1,2]studies the outage performance of dual-hop decode-and-forward (DF) cooperative systems with interference-limited relay in Nakagami-m fading environment. In[3,4,5,6]study the outage and bit-error performance in AF and DF cooperative system with interference-limited destination. And also the performance of cooperative networks with interference at relay and destination is studied in[7,8]. In[9], the author researches conventional and opportunistic relaying with interference at relay and destination, and obtain the upper bound of the error probability.

In this paper, we investigate the outage probability performance of the opportunity relay AF cooperative system with multiple co-channel interferes at relays and destination. And with the respect, the closed-form expression of the outage probability is derived. Through the analysis and simulation, the impact of co-channel interferes on the opportunity relay system can be presented.

\section{System Model}

We consider a communication system with multiple interferes at relays and destination. In this system, the source node $\mathrm{S}$ communicates with destination D can through two link, the direct link or the indirect link. Through the indirect link, the relay nodes amplify and forward the received information to the destination. This communication system is divided into two orthogonal time intervals. In first time slot, $\mathrm{S}$ sends its symbol $s_{0}$ to $R_{i}$ and $\mathrm{D}$. In second time slot, $R_{i}$ sends amplified symbol to D. We assume the additive white Gaussian noise (AWGN) terms of all links have zero mean and variance $N_{0}$

At the first phase, $\mathrm{S}$ sends its symbol $s_{0}$ to $\mathrm{D}$ and all the $L$ relays $\mathrm{R}$, the $L$ relays and destination node $\mathrm{D}$ received the faded and noisy source signal. At the same time, the $i^{\text {th }}$ relay received co-channel interfering signals from $N_{i}$ interfering nodes, the destination $\mathrm{D}$ also received 
interfering signals from $N_{D}$ interfering nodes. Therefore, the received signals at destination and the $i^{\text {th }}$ relay can be expressed as

$$
\begin{gathered}
y_{s d}=\sqrt{P_{0}} h_{s d} s_{0}+\sum_{k=1}^{N_{D}} \sqrt{P_{\mathrm{I}, k}} f_{s d_{k}} s_{k}+n_{0} \\
y_{r i}=\sqrt{P_{0}} h_{i} s_{0}+\sum_{j=1}^{N_{i}} \sqrt{P_{R_{i}}} f_{s r_{i, j}} g_{j}+n_{r i}
\end{gathered}
$$

Where $P_{0}$ is the transmit power of $\mathrm{S}, h_{s d}, h_{i}$ are the Rayleigh fading coefficients, $f_{s r_{i, j}}$ are the Rayleigh fading coefficients of the $j^{\text {th }}$ interference channel at the $i^{\text {th }}$ relay node. $f_{s d_{k}}$ are the Rayleigh fading coefficients of the $k^{\text {th }}$ interference channel at the destination. $s_{k}, g_{j}$ are the $k^{\text {th }}, j^{\text {th }}$ co-channel interfer's signal which can affect the destination and relay. $P_{I, k}, P_{R_{i}, j}$ are the energy of the $k^{\text {th }}$, $j^{t h}$ interference signal at destination and the $i^{\text {th }}$ relay respectively. $n_{0}, n_{r i}$ are the AWGN at the destination and the $i^{\text {th }}$ relay.

At the second phase, the relays will amplify and forward the received signal from the source node to the destination with a fixed gain $G_{i}$. So the destination's received signal can express as

$$
y_{R_{i}, D}=h_{r i, d} G_{i} y_{r i}+\sum_{l=1}^{M_{i}} \sqrt{P_{D_{i}}} f_{r_{i}, d_{l}} c_{i, l}+n_{r i, d}
$$

Where $h_{r i, d}$ is the Rayleigh fading coefficients, $f_{r_{i}, \mathrm{~d}}$ is the Rayleigh fading coefficients of the $l^{\text {th }}$ interference channel when the destination receives the $i^{\text {th }}$ relay's information. $c_{i, l}$ is the $l^{\text {th }}$ co-channel interfers' signal affects the $i^{\text {th }}$ relay signal at the destination. $n_{r i, d}$ is the AWGN at the destination. $G_{i}$ can be expressed as

$$
G_{i}=\sqrt{\frac{P_{r_{i}}}{P_{0}\left|h_{i}\right|^{2}+\sum_{j=1}^{N_{i}} P_{R_{i}}\left|f_{s r_{i, j}}\right|^{2}+N_{0}}}
$$

Where $P_{r_{i}}$ is the transmit power of the $i^{\text {th }}$ relay. In opportunity relaying cooperative system, the destination will receive the signal from only one link which has the highest SINR at the destination.

As a result, if the destination receives the signal from the direct link, the SINR at D can be expressed as 


$$
\gamma_{s}=\frac{P_{0}\left|h_{s d}\right|^{2}}{\sum_{k=1}^{N_{D}} P_{\mathrm{I}, k}\left|f_{s, d_{k}}\right|^{2}+N_{0}}
$$

If the destination receives the signal from the $i^{\text {th }}$ indirect link, the SINR at D can be expressed as

$$
\gamma_{b i}=\frac{P_{0} G_{i}{ }^{2}\left|h_{r i, d}\right|^{2}\left|h_{i}\right|^{2}}{G_{i}{ }^{2}\left|h_{r i, d}\right|^{2}\left(\sum_{j=1}^{N_{i}} P_{R_{i}}\left|f_{s r_{i, j}}\right|^{2}+N_{0}\right)+\sum_{l=1}^{M_{i}} P_{D, l}\left|f_{r_{i}, d_{l}}\right|^{2}+N_{0}}
$$

We define $\gamma_{0}=\frac{P_{0}\left|h_{s d}\right|^{2}}{N_{0}}, \gamma_{I}=\sum_{k=1}^{N_{D}} P_{\mathrm{I}, k} \frac{\left|f_{s, d_{k}}\right|^{2}}{N_{0}}, \gamma_{1, i}=\frac{P_{0}}{N_{0}}\left|h_{i}\right|^{2}, \quad \gamma_{2, i}=\frac{P_{r_{i}}}{N_{0}}\left|h_{r i, d}\right|^{2}, \quad \gamma_{R_{i}}=\sum_{j=1}^{N_{i}} P_{R_{i}, \mathrm{j}} \frac{\left|f_{s r_{i, j}}\right|^{2}}{N_{0}}$, $\gamma_{D_{i}}=\sum_{l=1}^{M_{i}} P_{\mathrm{D}, l} \frac{\left|f_{r_{i}, d_{l}}\right|^{2}}{\sigma_{d}^{2}}$, and $C_{i}=P_{0} \frac{\Omega_{s r_{i}}}{N_{0}}+\sum_{j=1}^{N_{i}} P_{R_{i}, \mathrm{j}} \frac{\Omega_{j}}{N_{0}}+1$. Where $\Omega_{s r_{i}}=\left.E|| h_{i}\right|^{2}\left|, \quad \Omega_{j}=E\right|\left|f_{s r_{i, j}}\right|^{2} \mid$, The expectation operator is $E\left[[]\right.$. Above all, $\gamma_{s}$ and $\gamma_{b i}$ can be expressed as

$$
\begin{aligned}
& \gamma_{s}=\frac{\gamma_{0}}{\gamma_{I}+1} \\
& \gamma_{b i}=\frac{\gamma_{1, i} \gamma_{2, i}}{\gamma_{2, i} \gamma_{R_{i}}+C_{i} \gamma_{D_{i}}}
\end{aligned}
$$

$h_{s d}, h_{i}, h_{r i, d} \mid$ are zero-mean complex Gaussian variables, so the probability density function (PDF) of the corresponding signal-to-noise ratio (SNR) $\gamma_{0}, \gamma_{1, i}, \quad \gamma_{2, i}(i=1,2 \ldots L)$, is all given as

$$
f_{\gamma}(\gamma)=\frac{1}{\bar{\gamma}} \exp \left(-\frac{\gamma}{\bar{\gamma}}\right)
$$

\section{System Performance Analysis}

From the second part we can know the destination will receive the source signal from only one link: direct link and indirect link which can be decided by the SINR at the destination in the opportunity relaying system. And the link with the highest SINR at the destination will be choose. So the outage probability of this system can be expressed as

$$
P_{\text {out }}=\operatorname{Pr}\left(\max \left(\gamma_{s}, \gamma_{b 1}, \gamma_{b 2}, \ldots \gamma_{b L}\right)<\gamma_{t h}\right)=F_{\gamma_{s}}\left(\gamma_{t h}\right) \prod_{i=1}^{L} F_{\gamma_{b i}}\left(\gamma_{t h}\right)
$$

For calculating Eq. 10, we need study the PDF of $\gamma_{R_{i}}, \gamma_{D_{i}}$ and $\gamma_{I}$. From paper [4], the PDF of $\gamma_{R_{i}}, \gamma_{D_{i}}$ and $\gamma_{I}$ all can be shown as

$$
f_{\gamma}(\gamma)=\sum_{i=1}^{\rho(A)} \sum_{j=1}^{\tau_{i}(A)} \chi_{i, j}(A) \frac{P_{i}^{-j}}{(j-1) !} \gamma^{j-1} \exp \left(-\frac{\gamma}{P_{i}}\right)
$$


Where $_{A}=\operatorname{diag}\left(\frac{P_{1}}{N_{0}^{2}} \Omega_{1}, \frac{P_{2}}{N_{0}^{2}} \Omega_{2}, \ldots, \frac{P_{\mathrm{N}}}{N_{0}^{2}} \Omega_{N}\right), \rho(A)$ denotes the number of distinct diagonal elements of $A, \quad P_{1}>P_{2}>\ldots>P_{[\rho(A)]}$ are the distinct diagonal elements in decreasing order, $\tau_{i}(A)$ is the multiplicity of $P_{i}$, and $\chi_{i, j}(A)$ is the $(i, \mathrm{j})$ th characteristic coefficient of $A$.

With the help of Eq. 9, Eq. 11 and the integral formula [10, Eq. (3.351.3)], $F_{\gamma_{s}}\left(\gamma_{t h}\right)$ can be formulated as

$$
\begin{aligned}
F_{\gamma_{s}}\left(\gamma_{t h}\right) & =\operatorname{Pr}\left(\frac{\gamma_{0}}{1+\gamma_{I}}<\gamma_{t h}\right) \\
& =1-\int_{0}^{\infty} \exp \left(-\frac{\left(1+\gamma_{I}\right)}{\bar{\gamma}_{0}} \gamma_{t h}\right) f_{\gamma_{I}}\left(\gamma_{I}\right) d \gamma_{I} \\
& =1-\exp \left(-\frac{\gamma_{t h}}{\bar{\gamma}_{0}}\right) \times \sum_{k=1}^{\rho(A)} \sum_{g=1}^{\tau_{k}(A)} \chi_{k, g}(A)\left(\frac{\gamma_{t h}}{\bar{\gamma}_{0}} P_{I, k}+1\right)^{-g}
\end{aligned}
$$

With the help of Eq. 9 and some manipulation, $F_{\gamma_{b}}\left(\gamma_{t h}\right)$ can be formulated as

$$
\begin{aligned}
F_{\gamma_{b i}}\left(\gamma_{t h}\right)=\operatorname{Pr}\left(\gamma_{b i}<\gamma_{t h}\right)=\operatorname{Pr}\left(=\frac{\gamma_{1, i} \gamma_{2, i}}{\gamma_{2, i} \gamma_{R_{i}}+C_{i} \gamma_{D_{i}}}<\gamma_{t h}\right) \\
=1-\int_{0}^{\infty} \exp \left(-\frac{\gamma_{t h}}{\bar{\gamma}_{1, i}} \gamma_{R_{i}}\right) f_{\gamma_{R_{i}}}\left(\gamma_{R_{i}}\right) d \gamma_{R_{i}} \\
\quad \times \int_{0}^{\infty} \int_{0}^{\infty} \exp \left(-C_{i} \gamma_{t h} \frac{\gamma_{D_{i}}}{\bar{\gamma}_{1, i} \gamma_{2, i}}\right) f_{\gamma_{2, i}}\left(\gamma_{2, i}\right) f_{\gamma_{D_{i}}}\left(\gamma_{D_{i}}\right) d \gamma_{2, i} d \gamma_{D_{i}} \\
=1-U_{1} \times U_{2}
\end{aligned}
$$

With the help of Eq. 9, Eq. 11 and the integral formula [10, Eq. (3.351.3)], $U_{1}$ can be formulated as

$$
\begin{aligned}
U_{1} & =\int_{0}^{\infty} \exp \left(-\frac{\gamma_{t h}}{\bar{\gamma}_{1, i}} \gamma_{R_{i}}\right) f_{\gamma_{R_{i}}}\left(\gamma_{R_{i}}\right) d \gamma_{R_{i}} \\
& =\sum_{m=1}^{\rho(B)} \sum_{n=1}^{\tau_{m}(B)} \frac{\chi_{\mathrm{m}, \mathrm{n}}(B) P_{R_{i}, \mathrm{~m}}^{-n}}{(n-1) !} \int_{0}^{\infty} \frac{\gamma_{R_{i}}^{n-1}}{\exp \left[\gamma_{R_{i}}\left(\frac{\gamma_{t h}}{\bar{\gamma}_{1, i}}+\frac{1}{P_{R_{i}, m}}\right)\right]^{d \gamma_{R_{i}}}} \\
& =\sum_{m=1}^{\rho(B)} \sum_{n=1}^{\tau_{m}(B)} \chi_{m, n}(B)\left(\frac{\gamma_{t h}}{\bar{\gamma}_{1, i}} P_{R_{i}, m}+1\right)^{-n}
\end{aligned}
$$

With the help of Eq. 9, Eq. 11 and the integral formula [10, Eq. (3.471.9)], $U_{2}$ can be formulated as 


$$
\begin{aligned}
U_{2} & =\int_{0}^{\infty} \int_{0}^{\infty} \exp \left(-C_{i} \gamma_{t h} \frac{\gamma_{D_{i}}}{\bar{\gamma}_{1, i} \gamma_{2, i}}\right) f_{\gamma_{2, i}}\left(\gamma_{2, i}\right) f_{\gamma_{D_{i}}}\left(\gamma_{D_{i}}\right) d \gamma_{2, i} d \gamma_{D_{i}} \\
& =\frac{1}{\bar{\gamma}_{2, i}} \int_{0}^{\infty} f_{\gamma_{D_{i}}}\left(\gamma_{D_{i}}\right) \int_{0}^{\infty} \exp \left(-C_{i} \gamma_{t h} \frac{\gamma_{D_{i}}}{\bar{\gamma}_{1, i} \gamma_{2, i}}-\frac{\gamma_{2, i}}{\bar{\gamma}_{2, i}}\right) d \gamma_{2, i} \\
& =\frac{2}{\bar{\gamma}_{2, i}} \int_{0}^{\infty} f_{\gamma_{D_{i}}}\left(\gamma_{D_{i}}\right) \sqrt{C_{i} \gamma_{t h} \frac{\bar{\gamma}_{2, i} \gamma_{D_{i}}}{\bar{\gamma}_{1, i}}} K_{1}\left(2 \sqrt{C_{i} \gamma_{t h} \frac{\gamma_{D_{i}}}{\bar{\gamma}_{1, i} \gamma_{2, i}}}\right) d \gamma_{D_{i}}
\end{aligned}
$$

Where $K_{n}(x)$ is the $n$ th-order modified Bessel function of the second kind [10, Eq. (3.471.9)]. Substituting Eq. 11 into Eq. 15, and after some algebraic manipulations, Eq. 15 can be calculated with the help of the integral formula [10, Eq. (6.631.3)], then it can obtained that

$$
U_{2}=\sum_{l=1}^{\rho(\mathrm{X}) \tau_{t}(\mathrm{X})} \sum_{t=1} \chi_{l, t}(\mathrm{X})(t !) \exp \left(\frac{C_{i} \gamma_{t h} P_{\mathrm{D}_{i}, l}}{4 \bar{\gamma}_{1, i} \bar{\gamma}_{2, i}}\right) W_{-t, \frac{1}{2}}\left(\frac{C_{i} \gamma_{t h} P_{\mathrm{D}_{i}, l}}{2 \bar{\gamma}_{1, i} \bar{\gamma}_{2, i}}\right)
$$

Where $W_{\lambda, \mu}(\chi)$ is the Whittaker function defined in [10 Eq.(9.222.1)], Substituting Eq. 14 and Eq. 15 into Eq. 13, it can be obtained that

$$
\begin{aligned}
F_{\gamma_{b i}}\left(\gamma_{t h}\right)= & 1-\sum_{m=1}^{\rho(B)} \sum_{n=1}^{\tau_{m}(B)} \chi_{m, n}(B)\left(\frac{\gamma_{t h}}{\bar{\gamma}_{1}} P_{R_{i}, m}+1\right)^{-n} \\
& \times \sum_{l=1}^{\rho(\mathrm{X}) \tau_{l}(\mathrm{X})} \sum_{t=1}^{-n} \chi_{l, t}(\mathrm{X})(t !) \exp \left(\frac{C_{i} \gamma_{t h} P_{\mathrm{D}_{i}, l}}{4 \bar{\gamma}_{1, i} \bar{\gamma}_{2, i}}\right) W_{-t, \frac{1}{2}}\left(\frac{C_{i} \gamma_{t h} P_{D_{i}, l}}{2 \bar{\gamma}_{1, i} \bar{\gamma}_{2, i}}\right)
\end{aligned}
$$

Then, Substituting Eq. 12 and Eq. 17 into Eq. 10, the outage probability of the AF cooperative diversity system with interferes can be given as Eq. 18

$$
\begin{aligned}
P_{\text {out }}= & {\left[1-\exp \left(-\frac{\gamma_{t h}}{\bar{\gamma}_{0}}\right) \times \sum_{k=1}^{\rho(A)} \sum_{l=1}^{\tau_{k}(A)} \chi_{k, l}(A)\left(\frac{\gamma_{t h}}{\bar{\gamma}_{0}} P_{I, k}+1\right)^{-l}\right] } \\
& \times \prod_{i=1}^{L}\left[1-\sum_{m=1}^{\rho(B) \tau_{m}(B)} \sum_{n=1}^{-(B)} \chi_{m, n}(B)\left(\frac{\gamma_{t h}}{\bar{\gamma}_{1, i}} P_{R_{i}, m}+1\right)^{-n} \times \sum_{l=1}^{\rho(\mathrm{X}) \tau_{l}(\mathrm{X})} \sum_{t=1} \chi_{l, t}(\mathrm{X})(t !) \exp \left(\frac{C_{i} \gamma_{t h} P_{D_{i}, l}}{4 \bar{\gamma}_{1, i} \bar{\gamma}_{2, i}}\right) W_{-t, \frac{1}{2}}\left(\frac{C_{i} \gamma_{t h} P_{D_{i}, l}}{2 \bar{\gamma}_{1, i} \bar{\gamma}_{2, i}}\right)\right]
\end{aligned}
$$

In this paper, the relay and destination are subjected to the equal-power interferes, so according to paper [3]: $P I=P_{\mathrm{I}, k} \Omega_{k} / N_{0}, \forall k=1,2 \ldots N_{I}, P_{R_{i}}=P_{R_{i}, j} \Omega_{j} / N_{0}, \forall j=1,2 \ldots N_{i}, P_{D_{i}}=P_{D_{i}, l} \Omega / N_{0}, \forall l=1,2 \ldots M_{i}$ an $\rho(A)=\rho(B)=\rho(\mathrm{X})=1, \quad \tau_{k}(A)=N_{\mathrm{I},} \tau_{m}(B)=N_{i}, \tau_{l}(\mathrm{X})=M_{i}, \chi_{1, \mathrm{~N}_{l}}(A)=1, \quad \chi_{1, k}(A)=0, k=1,2 \ldots N_{I}-1$ and the same as $\chi_{1, \mathrm{j}}(B), \chi_{1, l}(\mathrm{X}), \chi_{1, \mathrm{~N}_{i}}(B), \chi_{1, \mathrm{M}_{i}}(\mathrm{X})$.Therefore, Eq. 18 can be reduced to Eq. 19.

$$
P_{\text {out }}=\left[1-\exp \left(-\frac{\gamma_{t h}}{\bar{\gamma}_{0}}\right) \times\left(\frac{\gamma_{\text {th }}}{\bar{\gamma}_{0}} P_{\mathrm{I}}+1\right)^{-N_{I}}\right] \times \prod_{i=1}^{L}\left[1-\left(\frac{\gamma_{t h}}{\bar{\gamma}_{1, i}} P_{R_{i}}+1\right)^{-N_{i}} \times\left(M_{i} !\right) \exp \left(\frac{C_{i} \gamma_{t h} P_{\mathrm{D}_{i}}}{4 \bar{\gamma}_{1, i} \bar{\gamma}_{2, i}}\right) W_{-M_{i}, \frac{1}{2}}\left(\frac{C_{i} \gamma_{t h} P_{\mathrm{D}_{i}}}{2 \bar{\gamma}_{1, i} \bar{\gamma}_{2, i}}\right)\right]
$$

In this section,we will present the simulation results to show the effect of interferes for the AF cooperative diversity system. For simulating, it is assumed that the SIR in indirect link is defined as $\beta_{i}=E\left[\gamma_{1, i}\right] /\left(E\left[\gamma_{R_{i}}\right]+E\left[\gamma_{D_{i}}\right]\right), i=1,2 \cdots L$, the signal-to-interference ratio (SIR) in direct link is defined as $\beta_{0}=E\left[\gamma_{0}\right] / E\left[\gamma_{I}\right]$, and $10 \bar{\gamma}_{0}=\bar{\gamma}_{1, i}=\bar{\gamma}_{2, i}=\bar{\gamma}, \gamma_{t h}=5 d B, E\left[\gamma_{R_{i}}\right]=E\left[\gamma_{D_{i}}\right]$. Fig. 1 shows the 
outage performance as a function of the average $\operatorname{SNR}\left(\bar{\gamma}_{0}\right)$ for different number of interferes, where we set $L=1, \quad \beta_{0}=\beta_{i}=10 d B$ for $i=1, \cdots, L, N_{I}=N_{i}=M_{i}=N$. It shows that the outage performance appears worse when the number of interferes increased, and in the high SNR range the outage performance appears a floor effect for the impact of interferes. Fig. 2 shows the outage performance as a function of the average $\operatorname{SNR}\left(\bar{\gamma}_{0}\right)$ for relay selection in this opportunity relaying system with interferes, where we set $N=10, L=1,2,3,4, \beta_{0}=\beta_{i}=10 d B, i=1, \cdots, L$. It shows that the outage performance can be reduced effectively when the number of relay can be selected increases.

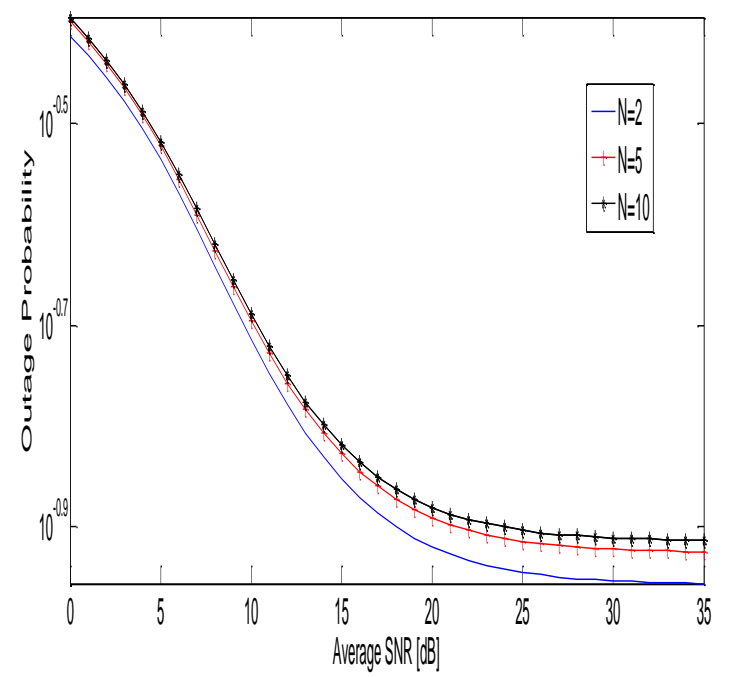

Figure 1. Outage probability as a function of the average $\operatorname{SNR}\left(\bar{\gamma}_{0}\right)$ for different number of interferes.

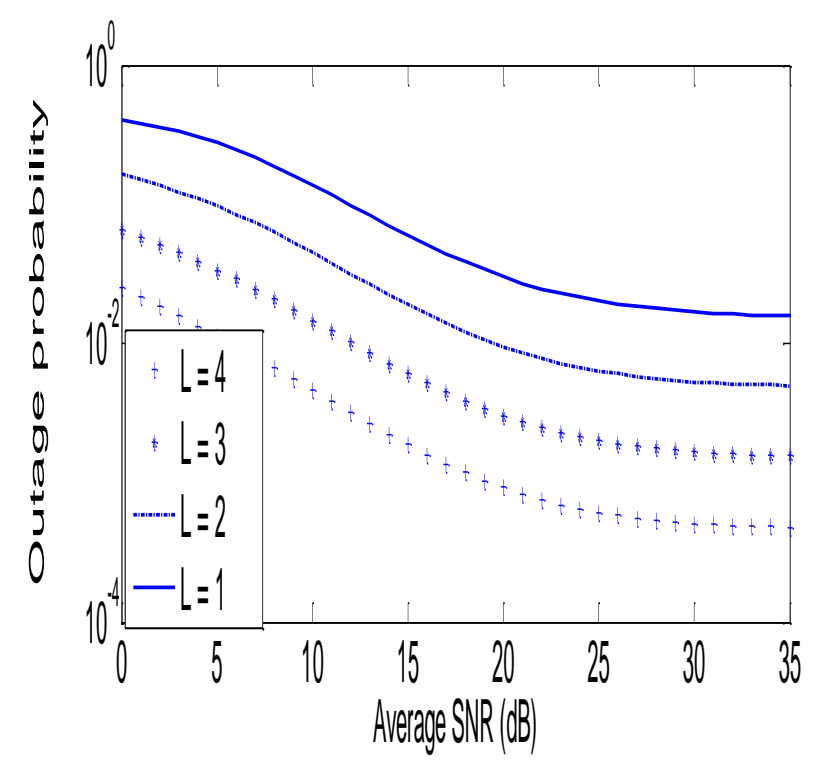

Figure 2. Outage probability against $S N R\left(\bar{\gamma}_{0}\right)$ of relay selection in this opportunity relaying system with interferes. 


\section{Conclusions}

In this paper, the outage probability performance of the opportunity relay AF cooperative system with multiple co-channel interferes at relays and destination is investigated. The closed expression of outage probability is derived. Through simulation, the result shows the outage performance will go to worse when the AF cooperative system is subjected to multiple interferes effect, but when this system increases the number of selected relays, the outage performance will be improved effectively.

\section{Acknowledgments}

This work is supported by project of the Fundamental Research Funds for the Central Universities (No. 31920140066)

\section{References}

[1] Daniel Benevides da Costa, Haiyang Ding ,Jianhua Ge. Interference-limited relaying transmissions in dual-hpo cooperative networks over Nakagami-m fading.IEEE communication LETTERS(2011), vol.15(5):503-505.

[2] Daniel, B. C. Haiyang, D. Michel, D. Y. and Jianhua Ge. Two-Way Relaying in Interference-Limited AF Cooperative Networks Over Nakagami-m Fading. In IEEE Trans.(2012), vol.61(8): .3766-3771.

[3] C. Zhong, S. Jin, and K.-K Wong, "Dual-hop systems with noisy relay and interference-limited destination," IEEE Trans. Commun.( 2010), vol. 58:764-768.

[4] Suraweera, H. A. Garg, H. K. and Nallanathan, A.Performance analysis of two hop amplify-and-forward systems with interference at the relay. In IEEE Communication. Letters(2010), vol.64:692-694.

[5] Suraweera, H. A. Michalopoulos, D. S. and Yuen, C. Performance analysis of fixed gain relay systems with a single interfere in Nakagami-m fading channels. IEEE Trans.(2012)vol. 61: 1457-1463.

[6] Amer M, M. Mamoun F, A. Rami, M. Ashraf H, S. BER Analysis in Relay-Based DF Cooperative Diversity Systems with Relay \& Destination Interferes..IEEE MIPRO (2015): 501-505.

[7] Wei Xu, Jianhua Zhang, Ping Zhang. Outage probability of two-hop fixed-gain relay with interference at relay and destination. IEEE COMMUNIATIONS LETTERS(2011), vol.15(6):608-610 .

[8] Rami, M. Mamoun F, A. Amer M, M. Ashraf H, S. Outage probability Analysis in Relay-Based DF Cooperative Diversity Systems with Relay \& Destination Interferes. IEEE MIPRO(2015): 506-511.

[9] Salama S. Ikki, P.Ubaidulla, Sonia Aissa. Performance study and optimization of cooperative diversity networks with Co-channel interference. IEEE THANSACTIONS ON WIRELESS COMMUNICATION(2014), vol.13(1):14-22.

[10] Gradshteyn, I. S. and Ryzhik, I. M. Table of Integrals, Series, and Products, 7th ed. Burlington, MA, USA: Academic Press.(2007). 